\title{
Isotropic-nematic spinodals of rigid long thin rodlike colloids by event-driven Brownian dynamics simulations
}

Yu-Guo Tao, W. K. den Otter, J. K. G. Dhont, and W. J. Briels

Citation: The Journal of Chemical Physics 124, 134906 (2006); doi: 10.1063/1.2180251

View online: https://doi.org/10.1063/1.2180251

View Table of Contents: http://aip.scitation.org/toc/jcp/124/13

Published by the American Institute of Physics

\section{Articles you may be interested in}

Brownian dynamics simulations of the self- and collective rotational diffusion coefficients of rigid long thin rods The Journal of Chemical Physics 122, 244903 (2005); 10.1063/1.1940031

The effect of steady flow fields on the isotropic-nematic phase transition of rigid rod-like polymers

The Journal of Chemical Physics 92, 792 (1990); 10.1063/1.458598

Shear-induced displacement of isotropic-nematic spinodals

The Journal of Chemical Physics 114, 10151 (2001); 10.1063/1.1372510

Event-driven Brownian dynamics for hard spheres

The Journal of Chemical Physics 126, 134109 (2007); 10.1063/1.2719190

Simulations of concentrated suspensions of rigid fibers: Relationship between short-time diffusivities and the long-time rotational diffusion

The Journal of Chemical Physics 123, 054908 (2005); 10.1063/1.1997149

Brownian dynamics algorithm for bead-rod semiflexible chain with anisotropic friction

The Journal of Chemical Physics 122, 084903 (2005); 10.1063/1.1848511

\section{PHYSICS TODAY}

WHITEPAPERS
ADVANCED LIGHT CURE ADHESIVES

READ NOW

Take a closer look at what these environmentally friendly adhesive systems can do
PRESENTED BY

Q8 MASTERBOND 


\title{
Isotropic-nematic spinodals of rigid long thin rodlike colloids by event-driven Brownian dynamics simulations
}

\author{
Yu-Guo Tao and W. K. den Otter ${ }^{\mathrm{a}}$ \\ Computational Biophysics, Faculty of Science and Technology, University of Twente, P.O. Box 217, \\ 7500 AE Enschede, The Netherlands \\ J. K. G. Dhont \\ Forschungszentrum Jülich, IFF/Weiche Materie, D-5225 Jülich, Germany \\ W. J. Briels \\ Computational Biophysics, Faculty of Science and Technology, University of Twente, P.O. Box 217, \\ 7500 AE Enschede, The Netherlands
}

(Received 11 January 2006; accepted 3 February 2006; published online 6 April 2006)

\begin{abstract}
The isotropic-nematic spinodals of solutions of rigid spherocylindrical colloids with various shape anisotropies $L / D$ in a wide range from 10 to 60 are investigated by means of Brownian dynamics simulations. To make these simulations feasible, we developed a new event-driven algorithm that takes the excluded volume interactions between particles into account as instantaneous collisions, but neglects the hydrodynamic interactions. This algorithm is applied to dense systems of highly elongated rods and proves to be efficient. The calculated isotropic-nematic spinodals lie between the previously established binodals in the phase diagram and extrapolate for infinitely long rods to Onsager's [Ann. N. Y. Acad. Sci. 51, 627 (1949)] theoretical predictions. Moreover, we investigate the shear induced shifts of the spinodals, qualitatively confirming the theoretical prediction of the critical shear rate at which the two spinodals merge and the isotropic-nematic phase transition ceases to exist. (C) 2006 American Institute of Physics. [DOI: 10.1063/1.2180251]
\end{abstract}

\section{INTRODUCTION}

Solutions of long and thin rigid rods interacting by excluded volume interactions, such as elongated colloidal particles and $f d$ viruses, ${ }^{1-3}$ exhibit lyotropic liquid crystalline phase behavior. At low concentrations the rods form a disordered isotropic phase, while at higher concentrations the interactions cause the rods to align along a director in a nematic phase. This phase still lacks translational order, which will appear at even higher concentrations. Upon increasing the rod number density, an isotropic solution will become thermodynamically unstable if the concentration crosses the isotropic-nematic spinodal (INS). Conversely, a nematic phase disorders into an isotropic state if the concentration is reduced below the nematic-isotropic spinodal (NIS). Outside these spinodals lie two binodals, i.e., the concentrations at which the coexisting isotropic and nematic phases of a phase separated system are in thermal equilibrium. In the 1940s, Onsager ${ }^{4}$ derived expressions for the spinodals and binodals of solutions of hard rods with infinite aspect ratios. The phase diagram of lyotropic rodlike systems has since been investigated extensively using theory, ${ }^{5-16}$ experiments, ${ }^{17,18}$ and more recently by computer simulations. ${ }^{19-21}$ For a review, we refer the reader to Vroege and Lekkerkerker. ${ }^{22}$

Our objective is to establish the spinodals of a solution of hard spherocylinders as a function of the aspect ratio of the rods. To this effect, we employ computer simulations to study the stability and dynamics of homogeneous ordered

\footnotetext{
${ }^{a)}$ Electronic mail: w.k.denotter@utwente.n
}

and disordered solutions in the vicinity of the spinodal concentrations. The dynamics of solvated rods is best simulated using Brownian dynamics (BD). At the high aspect ratios we are interested in, the hydrodynamic interactions between rods are less important ${ }^{23}$ than the excluded volume interactions, which are readily incorporated by means of a potential based on the overlap area. Since incorporating hydrodynamic interactions leads to complicated and slow programs, but does not affect the location of the spinodals, we here prefer a simpler and more approximate approach, leaving for future work the assessment of the importance of hydrodynamic interactions on the dynamics. Although recent studies ${ }^{24,25}$ of the self and collective rotational dynamics of rods show that this method works well for dilute systems, it unfortunately turns out that the interaction potentials cause unsurmountable difficulties when performing prolonged simulations of the dense and highly elongated rods needed for a stable nematic phase. We therefore introduce here an event-driven Brownian dynamics algorithm, combining the dynamics equations of traditional fixed time step BD with the collision-based time step commonly used in simulations of hard bodies. The resulting algorithm proves efficient at simulating concentrated solutions of rods, and displays a stable nematic phase for elongated rods. The notoriously slow process of spontaneous ordering of an isotropic system above the INS takes between a week and a month on a desktop personal computer for the densities and high aspect ratios employed here.

Having direct access to the dynamics of the rods also allows us to investigate the effect of shear flow on the phase diagram. Under shear the rods have a propensity to align 
along the flow direction, both in the isotropic and in the nematic phase, thus decreasing the spinodal concentrations. ${ }^{23,26-32}$ At sufficiently high shear rates the spinodals are predicted to terminate at a coalescence point, eliminating the phase transition between ordered and disordered states.

This paper is organized as follows: The event-driven Brownian dynamics algorithm is described in detail in the next section, followed by a validation against a traditional $\mathrm{BD}$ program at the dilute and semidilute concentrations accessible to the latter. In Sec. III A we investigate the density dependence of the self and collective rotational diffusion coefficients. The spinodals as functions of the aspect ratio are presented in Sec. III B, and in Sec. III C we briefly discuss the shear-induced shift of the spinodals. Finally, we summarize our conclusions in Sec. IV.

\section{SIMULATION METHOD}

\section{A. Event-driven algorithm}

In this section we briefly describe the algorithm employed in event-driven Brownian dynamics simulations. The Brownian particles, in this case rigid long and thin colloids, are suspended in a Newtonian liquid with a stationary simple shear velocity field $\mathbf{V}$

$$
\mathbf{V}(\mathbf{r})=\boldsymbol{\Gamma} \cdot \mathbf{r},
$$

with

$$
\boldsymbol{\Gamma}=\dot{\boldsymbol{\Gamma}} \text { and } \hat{\boldsymbol{\Gamma}}=\left(\begin{array}{lll}
0 & 1 & 0 \\
0 & 0 & 0 \\
0 & 0 & 0
\end{array}\right)
$$

Here $\mathbf{r}$ is a position, $\dot{\gamma}$ is the shear rate, and $\boldsymbol{\Gamma}$ is the transposed velocity gradient tensor. This velocity field corresponds to a flow in the $x$ direction, with a gradient in the $y$ direction.

The forces and torques acting on the Brownian particles have three origins: potential, friction, and random. The first is due to the excluded volume interactions between solute particles; the second is caused by the velocity and angular velocity of the particle relative to the flow field; the last one is due to thermal collisions between particles and solvent molecules. For a very long and thin rod, due to its symmetry, the rotational motion around the cylindrical axis does not couple to the remaining degrees of freedom and may be neglected. Generally, the Brownian motion of particles can be described, on the Smoluchowski or diffusive time scale, by stochastic differential equations (SDEs), also known as Langevin equations. For our long thin rods in a shear flow, these equations are

$$
\begin{aligned}
\mathbf{r}(t+\delta t)= & \mathbf{r}(t)+\boldsymbol{\Gamma} \cdot \mathbf{r}(t) \delta t+\Xi^{-1}(t) \cdot \mathbf{F}_{S}(t) \delta t+\delta \mathbf{r}(t), \\
\hat{\mathbf{u}}(t+\delta t)= & \hat{\mathbf{u}}(t)+[\hat{\mathbf{I}}-\hat{\mathbf{u}}(t) \hat{\mathbf{u}}(t)] \cdot \boldsymbol{\Gamma} \cdot \hat{\mathbf{u}}(t) \delta t \\
& +\frac{1}{\gamma_{r}} \mathbf{T}_{S}(t) \times \hat{\mathbf{u}}(t) \delta t+\delta \hat{\mathbf{u}}(t) .
\end{aligned}
$$

Here, $\mathbf{r}$ represents the center of mass position of a rod, $\hat{\mathbf{u}}$ is a unit vector along the axial direction of the $\operatorname{rod}, \mathbf{F}_{S}$ and $\mathbf{T}_{S}$ denote the systematic force and torque, respectively, $\hat{\mathbf{I}}$ is the unit tensor, and $\delta t$ is the time step used in the simulations. In Eq. (3), the translational friction tensor $\Xi$ is orientation dependent:

$$
\Xi=\gamma_{\|} \hat{\mathbf{u}} \hat{\mathbf{u}}+\gamma_{\perp}(\hat{\mathbf{I}}-\hat{\mathbf{u}} \hat{\mathbf{u}}),
$$

where $\gamma_{\|}$and $\gamma_{\perp}$ are the translational friction coefficients for motions parallel and perpendicular to the axis of the rod, respectively, while in Eq. (4) $\gamma_{r}$ denotes the rotational friction coefficient. ${ }^{33,34}$ From hydrodynamics, the following expressions can be derived for long and thin rods of length $L$ and diameter $D$ :

$$
\begin{aligned}
& \gamma_{r}=\frac{\pi \eta_{s} L^{3}}{3 \ln (L / D)}, \\
& \gamma_{\|}=\frac{2 \pi \eta_{s} L}{\ln (L / D)}, \\
& \gamma_{\perp}=2 \gamma_{\|},
\end{aligned}
$$

where $\eta_{s}$ denotes the viscosity of the solvent. The random displacement and reorientation $\delta \mathbf{r}(t)$ and $\delta \hat{\mathbf{u}}(t)$, respectively, are sampled from Gaussian distributions with zero mean and variances

$$
\langle\delta \mathbf{r}(t) \delta \mathbf{r}(t)\rangle=2 k_{B} T \Xi^{-1}(t) \delta t
$$

$$
\langle\delta \hat{\mathbf{u}}(t) \delta \hat{\mathbf{u}}(t)\rangle=2 k_{B} T \frac{1}{\gamma_{r}}(\hat{\mathbf{I}}-\hat{\mathbf{u}} \hat{\mathbf{u}}) \delta t,
$$

with $k_{B} T$ the thermal energy. The brackets indicate canonical ensemble averages. In the computer program, $\delta \mathbf{r}(t)$ and $\delta \hat{\mathbf{u}}(t)$ are treated in the same way as mentioned in our previous paper: $^{24}$

$$
\begin{aligned}
\delta \mathbf{r}(t)= & G_{1} \sqrt{\frac{2 k_{B} T \delta t}{\gamma_{\|}}} \hat{\mathbf{u}}+G_{2} \sqrt{\frac{2 k_{B} T \delta t}{\gamma_{\perp}}} \frac{\hat{\mathbf{u}} \times \hat{\mathbf{e}}_{x}}{\left|\hat{\mathbf{u}} \times \hat{\mathbf{e}}_{x}\right|} \\
& +G_{3} \sqrt{\frac{2 k_{B} T \delta t}{\gamma_{\perp}}} \frac{\hat{\mathbf{u}} \times\left(\hat{\mathbf{u}} \times \hat{\mathbf{e}}_{x}\right)}{\left|\hat{\mathbf{u}} \times\left(\hat{\mathbf{u}} \times \hat{\mathbf{e}}_{x}\right)\right|}, \\
\delta \hat{\mathbf{u}}(t)= & G_{4} \sqrt{\frac{2 k_{B} T \delta t}{\gamma_{r}}} \frac{\hat{\mathbf{u}} \times \hat{\mathbf{e}}_{x}}{\left|\hat{\mathbf{u}} \times \hat{\mathbf{e}}_{x}\right|} \\
& +G_{5} \sqrt{\frac{2 k_{B} T \delta t}{\gamma_{r}}} \frac{\hat{\mathbf{u}} \times\left(\hat{\mathbf{u}} \times \hat{\mathbf{e}}_{x}\right)}{\left|\hat{\mathbf{u}} \times\left(\hat{\mathbf{u}} \times \hat{\mathbf{e}}_{x}\right)\right|},
\end{aligned}
$$

where $G_{1-5}$ are Gaussian random numbers with unit variance.

Note that the above algorithm will gradually change the length of the unit vector $\hat{\mathbf{u}}$. There are two approaches to correct for this artifact: one can reset the length of the rods at the end of every time step by correcting either along the initial or along the final direction of the rod. This point has been thoroughly investigated by Cobb and Butler, ${ }^{25}$ who found that the first method is the proper way. We have tested both methods in our simulations, and found that for the time step used here, there was no significant difference between the two approaches. 
Equations (3), (4), (9), and (10) constitute the equations of motion used in this paper. We shall now prove that they give rise to the $\mathrm{N}$-particle Smoluchowski equation,

$$
\begin{aligned}
\frac{\partial P}{\partial t}= & \sum_{j=1}^{N}\left\{\frac{3}{4} D_{t} \nabla_{j} \cdot\left(\hat{\mathbf{I}}+\hat{\mathbf{u}}_{j} \hat{\mathbf{u}}_{j}\right) \cdot\left[\nabla_{j} P+\beta P \nabla_{j} \Phi\right]\right. \\
& -\nabla_{j} \cdot\left[P \boldsymbol{\Gamma} \cdot \mathbf{r}_{i}\right]+D_{r} \hat{\mathcal{R}}_{j} \cdot\left[\hat{\mathcal{R}}_{j} P+\beta P \hat{\mathcal{R}}_{j} \Phi\right] \\
& \left.-\hat{\mathcal{R}}_{j} \cdot\left[P \hat{\mathbf{u}}_{j} \times\left(\boldsymbol{\Gamma} \cdot \hat{\mathbf{u}}_{j}\right)\right]\right\},
\end{aligned}
$$

which is considered to be the fundamental equation of motion ${ }^{23,34,35}$ for the probability density function $P$ of finding the system at time $t$ in a state $z=\left\{z_{1}, \ldots, z_{N}\right\}$ with $z_{i}=\left\{\mathbf{r}_{i}, \hat{\mathbf{u}}_{i}\right\}$, given that it was in the state $z_{0}$ at time zero. Here, $\nabla_{j}$ denotes the gradient with respect to the position of particle $j$ and $\hat{\mathcal{R}}_{j} \equiv \hat{\mathbf{u}}_{j} \times \nabla_{\hat{\mathbf{u}}_{j}}$, where $\nabla_{\hat{\mathbf{u}}_{j}}$ is the gradient with respect to the orientation of particle $j$. Furthermore, the diffusion coefficients $D_{t}$ and $D_{r}$ are given by

$$
\begin{aligned}
D_{t} & =\frac{D_{\|}+2 D_{\perp}}{3}=\frac{k_{B} T \ln (L / D)}{3 \pi \eta_{s} L}, \\
D_{r} & =\frac{3 k_{B} T \ln (L / D)}{\pi \eta_{s} L^{3}} .
\end{aligned}
$$

The proof that we are going to give should be considered to be the decisive motivation for choosing the above equations of motion. The equivalence of the Brownian dynamics equations of motion and the Smoluchowski equation in the absence of shear flow has been derived in Appendix B of Ref. 24. When shear is taken into account, an additional term will appear in each of the expressions of the first moments described by Eq. (B8) of Ref. 24:

$$
\begin{aligned}
& \langle\Delta \mathbf{r} ; \delta t\rangle=-\Xi^{-1}(t) \cdot \nabla \Phi(t) \delta t+\boldsymbol{\Gamma} \cdot \mathbf{r}(t) \delta t, \\
& \langle\Delta \hat{\mathbf{u}} ; \delta t\rangle=\frac{1}{\gamma_{r}} \hat{\mathbf{u}}(t) \times \hat{\mathcal{R}} \Phi(t) \delta t+[\hat{\mathbf{I}}-\hat{\mathbf{u}}(t) \hat{\mathbf{u}}(t)] \cdot \boldsymbol{\Gamma} \cdot \hat{\mathbf{u}}(t) \delta t .
\end{aligned}
$$

Introducing these expressions into Eq. (B9) of Ref. 24 and using

$$
\begin{aligned}
& (\hat{\mathbf{I}}-\hat{\mathbf{u}} \hat{\mathbf{u}}) \cdot \nabla_{\hat{\mathbf{u}}}=-\hat{\mathbf{u}} \times \hat{\mathcal{R}}, \\
& \oint d \hat{\mathbf{u}} \mathbf{A}(\hat{\mathbf{u}}) \cdot \hat{\mathcal{R}} F(z)=-\oint d \hat{\mathbf{u}}(\hat{\mathcal{R}} \cdot \mathbf{A}(\hat{\mathbf{u}})) F(z),
\end{aligned}
$$

where $F$ and $\mathbf{A}$ are an arbitrary function and vector field, respectively, we end up with two additional terms inside the curly brackets on the right-hand side of Eq. (B11) of Ref. 24:

$$
\begin{aligned}
&-\nabla \cdot \boldsymbol{\Gamma} \cdot \mathbf{r} P\left(z, z_{0} ; t\right) \\
& \quad+\hat{\mathcal{R}} \cdot\left\{[(\hat{\mathbf{I}}-\hat{\mathbf{u}} \hat{\mathbf{u}}) \cdot \boldsymbol{\Gamma} \cdot \hat{\mathbf{u}}] \times \hat{\mathbf{u}} P\left(z, z_{0} ; t\right)\right\} .
\end{aligned}
$$

Summing over all particles on the right-hand side of Eq. (B11) in Appendix B of Ref. 24, we obtain the $N$-particle Smoluchowski equation Eq. (13) given above.
In the case of dilute solutions, the systematic forces $\mathbf{F}_{S}$ and torques $\mathbf{T}_{S}$ are hardly important and can be neglected. However, in the case of concentrated solutions, mutual interactions between rods play a crucial role and the systematic forces $\mathbf{F}_{S}$ and torques $\mathbf{T}_{S}$ need to be taken into account. In our previous model, $\mathbf{F}_{S}$ and $\mathbf{T}_{S}$ were derived from an excluded volume potential $\Phi$, which was chosen to be proportional to the overlap volume between two interacting rods. This model was successfully applied to measure the self and collective rotational diffusion coefficients in dilute and semidilute rod solutions. ${ }^{24}$ But when focusing on concentrated systems of hard rods, some disadvantages of this model appear. As a result of the random displacements and reorientations, sometimes considerable overlaps and even crossings cannot be prevented, irrespective of the smallness of the time step $\delta t$. In order to achieve simulations of infinitely hard rod systems, a new simulation technique had to be developed.

In our new simulation program, the excluded volume potential and its corresponding systematic forces and torques have been eliminated in favor of an event-driven technique, ${ }^{36}$ inspired by the celebrated molecular dynamics simulations of hard spheres by Alder and Wainwright, ${ }^{37}$ which have been extended to molecular dynamics simulations of infinitely thin rods by Frenkel and Maguire. ${ }^{38}$ Between collisions, every rod performs a Brownian motion, independent of the other rods. At a collision this motion changes abruptly. If the linear and angular velocities of the colliding rods are known, it is straightforward to solve the impulsive collision analytically (including the two constraints on the lengths of the rod). In the overdamped case, when Brownian dynamics applies, the system looses its memory of the initial velocities long before its configuration has changed appreciably. In this case the velocities can be eliminated altogether and the time step is adjusted to the rate of change of the system configurations. Random displacements and reorientations are applied to the particles as, for example, in Eqs. (11) and (12). If the particle finds itself near a wall or any other obstacle, for example, a companion particle, these random contributions have to be modified in a way which is known analytically only in a few simple cases with spherical particles. ${ }^{39-41}$ It has been found, however, that in these cases no noticeable differences occur if the particle is moved in the usual way up to the point of contact with the obstacle and new random displacements, away from overlap, are chosen to complete the time step. Several alternative approximate methods have been suggested to remove possible overlaps at time $t+\delta t$, all being more or less equally efficient. ${ }^{42-47}$

We propose to use a similar method in the case of Brownian dynamics simulations of rodlike colloids, i.e., we use the random forces and torques at time $t$ to advance two colliding rods up to the time of contact $t+\lambda \delta t$ with $0<\lambda$ $<1$, according to an obvious modification of the original equations of motion,

$$
\mathbf{r}_{i}(t+\lambda \delta t)=\mathbf{r}_{i}(t)+\boldsymbol{\Gamma} \cdot \mathbf{r}_{i}(t) \lambda \delta t+\delta \mathbf{r}(t) \sqrt{\lambda},
$$




$$
\begin{aligned}
\hat{\mathbf{u}}_{i}(t+\lambda \delta t)= & \hat{\mathbf{u}}_{i}(t)+\left[\hat{\mathbf{I}}-\hat{\mathbf{u}}_{i}(t) \hat{\mathbf{u}}_{i}(t)\right] \\
& \cdot \boldsymbol{\Gamma} \cdot \hat{\mathbf{u}}_{i}(t) \lambda \delta t+\delta \hat{\mathbf{u}}(t) \sqrt{\lambda} .
\end{aligned}
$$

At contact, new random displacement $\delta \mathbf{r}^{\prime}(t)$ and reorientation $\delta \hat{\mathbf{u}}^{\prime}(t)$ are sampled according to Eqs. (11) and (12), and

$$
\begin{aligned}
\mathbf{r}_{i}(t+\delta t)= & \mathbf{r}_{i}(t+\lambda \delta t)+\boldsymbol{\Gamma} \cdot \mathbf{r}_{i}(t+\lambda \delta t) \delta t(1-\lambda) \\
& +\delta \mathbf{r}^{\prime}(t) \sqrt{1-\lambda}, \\
\hat{\mathbf{u}}_{i}(t+\delta t)= & \hat{\mathbf{u}}_{i}(t+\lambda \delta t)+\left[\hat{\mathbf{I}}-\hat{\mathbf{u}}_{i}(t+\lambda \delta t) \hat{\mathbf{u}}_{i}(t+\lambda \delta t)\right] \\
& \cdot \boldsymbol{\Gamma} \cdot \hat{\mathbf{u}}_{i}(t+\lambda \delta t) \delta t(1-\lambda)+\delta \hat{\mathbf{u}}(t) \sqrt{1-\lambda,}
\end{aligned}
$$

are used to complete the time step.

In order to advance the system from time $t$ to time $t+\delta t$, we perform the following steps:

(1) At time $t$, a list of all possible collision pairs is compiled consisting of those pairs whose shortest distance is smaller than a certain distance criterion, say, $r_{\text {cut }}$. Shortest distance here means the shortest possible distance obtained by moving one point along the surface of one rod and another point along the surface of the other rod. This distance can conveniently be calculated for cylinders capped with hemispheres of equal radius. By regarding the oblong rods as being constructed from several segments and using a segment based grid and link list, this step may be accelerated appreciably.

(2) All rods are advanced from $t$ to $t+\delta t$ according to free Brownian motions. The list of possible collision pairs is completed by adding those pairs whose shortest distance is smaller than $r_{\text {cut }}$ at this new time $t+\delta t$. The criterion $r_{\text {cut }}$ is the maximum separation between two rods that still allows for a collision to occur within a time lapse of $\frac{1}{2} \delta t$ or, in other words, the maximum distance that can occur between two rods during a time lapse of $\frac{1}{2} \delta t$ after initial contact; hence if a collision takes place between $t$ and $t+\delta t$ the distance between the rods must be less than $r_{\text {cut }}$ at $t$ and/or at $t+\delta t$. Clearly, $r_{\text {cut }}$ is a function of the viscosity, time step, etc. With a time step of $0.5 \mu \mathrm{s}$, it proves that using the criterion $r_{\text {cut }}=D$ is always safe for preventing overlaps between rods.

(3) For all pairs in the list, the time of first contact is determined by interpolation of the already chosen Brownian displacements and reorientations. To this end, the time interval is subdivided into 100 smaller intervals and configurations are attributed to the end points of these intervals $l$ using the original random numbers and the time lapse $l \delta t / 100$ since the beginning of the time step. Note that this implies that the trajectories are assumed to be rectilinear in configuration space, but that the distance traveled along these trajectories varies with the square root of the time lapse, $\sqrt{l \delta t / 100}$. Finally, for each pair the time of first contact is detected and the pairs are ordered according to increasing time of first contact.

(4) The pair with the shortest time of first contact is advanced up to contact. Next, new random displacements

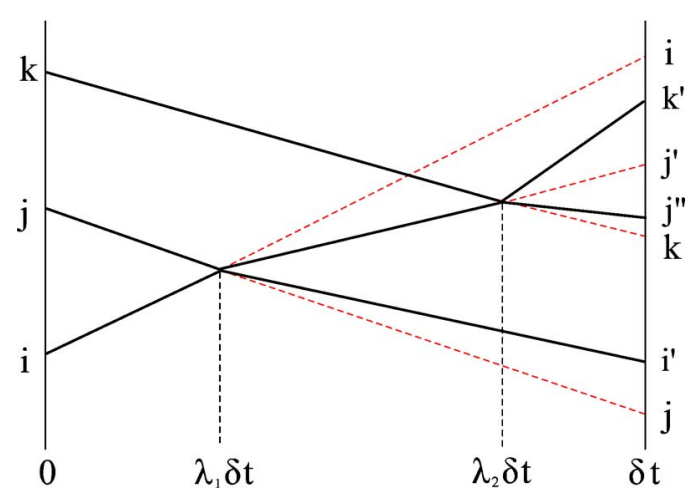

FIG. 1. A cartoon of the trajectories of three interacting rods during one time step $\delta t$. On the left-hand side, $i, j$, and $k$ mark the start positions of the rods. On the right-hand side, $i, j$, and $k$ denote the prospective positions of the rods at the end of the step for the given initial set of random forces and torques. At the collision moments $\lambda_{1} \delta t$ and $\lambda_{2} \delta t$ new random forces and torques are drawn for the two colliding particles. The resulting modified final positions are indicated by (double) primed indices. The solid lines denote the traversed trajectories; dotted lines are extrapolations.

and reorientations are chosen for this pair such that the rods separate initially and the table of collision pairs is updated. New times of first contact are calculated for all pairs in the list which involve one of the two rods of the last collision and step 4 is repeated until all possible collisions within the given time step have been exhausted. This way, a rod may collide several times per time step, as illustrated by rod $j$ in Fig. 1, and it is even possible for two rods to collide repeatedly with each other in one step.

A comment as to the chosen method is in order here. As we already mentioned, the exact propagator for colliding Brownian particles is known for a few cases only. For pointlike particles near a flat wall, each particle which after a trial step ends in the forbidden area should be mirrored in the wall. For colliding rods no exact solution is known; the reflection method proposed by Doi et al. ${ }^{48}$ in which a particle retraces its path after a collision, can only be considered a very approximate solution. Harlen et al. ${ }^{49}$ prevented rods from crossing by introducing a normal constraint force between the rods, whose strength is determined by the condition that the nearest distance cannot drop below the rod diameter. These procedures are only correct in the limit of very small time steps, in which case the number of regular Brownian steps by far exceeds the number of (approximate) collision steps. Therefore, we have chosen to subdivide the large main time step into smaller time steps for each colliding pair, assuming that the smallest time step still exceeds the contact time. The latter assumption is perhaps less realistic at high shear rates, where the flow may force the rods to slip along one another.

The simulation system in the present work is based on the experimental data of the $f d$ virus. ${ }^{1}$ The simulation box is cubic and contains $N=c L \varphi / D$ rods, with $c=500$ for $L / D$ $\leqslant 30$ and $c=1000$ for $L / D \geqslant 60$, of diameter $D=14.8 \mathrm{~nm}$. Here $\varphi$ denotes volume fraction, defined as $\varphi=\frac{1}{4} \pi n D^{2} L$ for hard rods with $n$ the number density, and $L \varphi / D$ is refered to as the scaled volume fraction. The aspect ratios $L / D$ range 


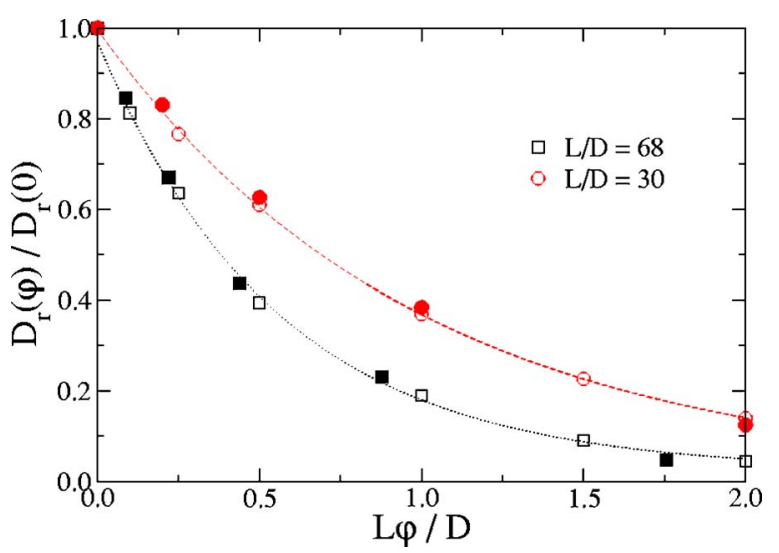

FIG. 2. The rotational self-diffusion coefficients of rigid rods with aspect ratios of $L / D=30$ and 68 as functions of the scaled volume fraction $L \varphi / D$. The open symbols denote results calculated with the new event-driven algorithm for hard rods, while the solid symbols are obtained by our previous algorithm in which the interaction potential between rods was proportional to their overlap volume.

from 10 to 68 ; hence the lengths of the rods range from $L=0.148 \mu \mathrm{m}$ to $L=0.888 \mu \mathrm{m}$. Water is used as the solvent, with viscosity $\eta_{s}=10^{-3} \mathrm{~Pa}$. The temperature is $300 \mathrm{~K}$. The efficiency of the above described algorithm is largely determined by the chosen time step. At a low value of $\delta t$ there will be few collisions, but a large number of time steps are needed; for high $\delta t$ the length of the collision list grows rapidly, and we might even need smaller interpolation steps to determine the times of first contact. The optimum value of $\delta t$ will vary with the system studied. To make sure that during one time step the rods will not move or rotate too much, the time step $\delta t$ is chosen in such a way that the maximum random displacement is smaller than one-tenth of the thickness of the rod and so that the maximum random reorientation will not be larger than $2 D / 10 L$. We thus arrive at a time step $\delta t=0.5 \mu$ s used for all simulations in this paper, which is ten times larger than that used in our previous simulations ${ }^{24}$ with soft interactions between rods. Notice that this is still two orders of magnitude smaller than the time step for which Cobb and Butler ${ }^{25}$ discern a difference between the two approaches to conserve the length of the unit vector $\hat{\mathbf{u}}$.

\section{B. Validation of the algorithm}

In order to test the new algorithm, Brownian dynamics simulations were carried out measuring the rotational selfdiffusion coefficients $D_{r}(\varphi)$ of dilute and semidilute solutions of rods. We measured these coefficients by calculating

$$
\langle\hat{\mathbf{u}}(t) \cdot \hat{\mathbf{u}}(0)\rangle=\exp \left\{-2 D_{r}(\varphi) t\right\} .
$$

The results are presented in Fig. 2 as a function of $L \varphi / D$ for two values of $L / D$ together with similar results obtained with our previous program. ${ }^{24}$ As is clear from this figure, the results obtained with the two programs are in perfect agreement with each other. Besides this agreement, preliminary simulations of the dynamics of dense solutions of rigid rods in shear flow have revealed ${ }^{50}$ periodic motions whose periods are in good agreement with experimental results. We therefore consider our new algorithm to correctly describe the dynamics of Brownian rods, even when applied to dense systems.

\section{Start configurations}

The orientational order is usually measured by the scalar order parameter $P_{2}$, defined as $P_{2}=(3 \lambda-1) / 2$, where $\lambda$ is the largest eigenvalue of the orientational order parameter tensor

$$
\mathbf{S}=\frac{1}{N} \sum_{i=1}^{N} \hat{\mathbf{u}}_{i} \hat{\mathbf{u}}_{i} .
$$

Hence, $P_{2}$ is zero in an isotropic state and becomes unity in a well aligned nematic state. The director $\hat{\mathbf{n}}$, given by the eigenvector corresponding to $\lambda$, points along the average direction of the rods. Investigations of the motions that $\hat{\mathbf{n}}$ exhibits in a shear flow will be published elsewhere. ${ }^{50,51}$

It is relatively easy to construct an initially perfectly aligned state by randomly placing parallel rods in the box, rejecting those rods that overlap with a previously accepted rod. This procedure is repeated until the desired number of rods is reached. An initially isotropic box is made in exactly the same way, selecting both positions and orientations of every inserted rod at random. This production becomes increasingly challenging with rising volume fraction and aspect ratio, as more and more rods are being rejected. In practice, we find that scaled volume fractions $L \varphi / D$ of $3,3.5$, and 4 are attainable this way for $L / D=10,15$, and $>20$, respectively. Williams and Philipse ${ }^{52}$ have shown that the theoretical maximum is given by $L \varphi / D=5.1$, which lies well above our scaled volume fractions. The isotropy of the produced boxes is tested by confirming that the order matrix is isotropic, $S_{i j}(0) \approx \frac{1}{3} \delta_{i j}$, and by the absence of domains under visual inspection.

\section{RESULTS AND DISCUSSIONS}

In this section, we describe the results of our simulations of semidilute and dense systems of rodlike colloids. First, we address the scaling of the rotational self-diffusion with density. In the remaining part of this section, we study the spinodals of the isotropic-nematic phase transition.

\section{A. Rotational diffusion coefficients}

In Fig. 3, we have plotted the rotational diffusion coefficients as a function of $L \varphi / D$ for various aspect ratios. At the relatively low volume fractions to which this figure applies, the rotational self-diffusion coefficients are virtually equal to the collective rotational diffusion coefficients (vide infra), which by Dhont and Briels were predicted to be linear in $L \varphi / D$. The marked difference between the simulation results and the theoretical prediction must be due to the neglect of dynamic correlations in the latter. An alternative, more phenomenological analysis based on the tube concept has been presented by Doi and Edwards, ${ }^{53,54}$ which was then confirmed by Monte Carlo simulations ${ }^{48}$ on systems of rods with zero diameter. According to their theory, the rotational self-diffusion coefficients in the semidilute regime should scale as 


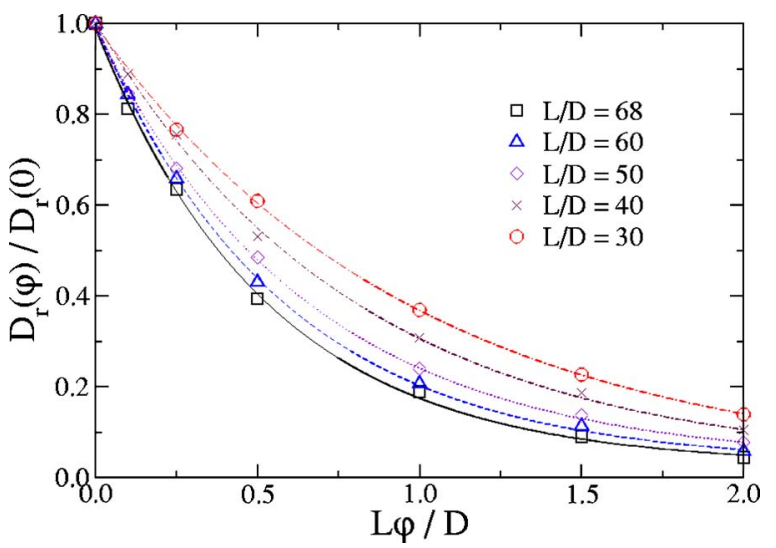

FIG. 3. The rotational self-diffusion coefficients of rigid rods with various aspect ratios as functions of the scaled volume fraction.

$$
D_{r}(\varphi)=\beta D_{r}(0)\left(n L^{3}\right)^{-2},
$$

where $\beta$ is some numerical parameter. In Fig. 4, we plot our results as a function of $n L^{3}$ on a log-log scale. It is clearly seen from this figure that with increasing values of $L / D$, the curves approach a limiting master curve with a slope equal to -2 , thereby confirming the prediction of Doi and Edwards. Similar evidence has recently been published by Cobb and Butler. $^{25}$

The collective rotational diffusion coefficient describes the decay back to zero of a small perturbation $\delta \mathbf{S}(t)=\mathbf{S}(t)$ $-\frac{1}{3} \hat{\mathbf{I}}$ to the isotropic state. This can be obtained by starting with an aligned state and picking up the late stage behavior. For volume fractions where the decay becomes very slow, this cannot be done any more and we resort to invoke Onsager's regression hypothesis, ${ }^{55,56}$ which states that time dependent fluctuations in a stable or metastable state decay according to the macroscopic laws, i.e., in the present case,

$$
\langle\delta \mathbf{S}(t) \delta \mathbf{S}(0)\rangle=\langle\delta \mathbf{S}(0) \delta \mathbf{S}(0)\rangle \exp \left\{-6 D_{r}^{\text {coll }}(\varphi) t\right\},
$$

where $D_{r}^{\text {coll }}(\varphi)$ is the collective rotational diffusion coefficient.

In Fig. 5, we present both the self and collective rotational diffusion coefficients as functions of $L \varphi / D$ for $L / D$ $=50$. As mentioned before, ${ }^{24}$ at lower volume fractions, both

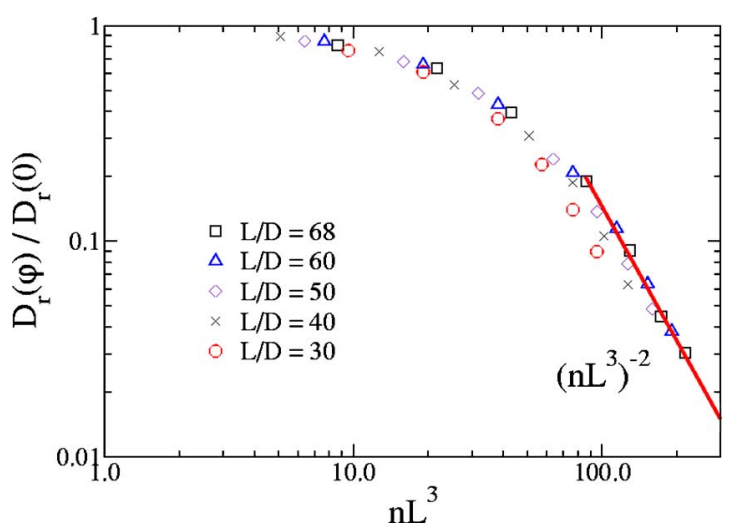

FIG. 4. The rotational self-diffusion coefficients of rigid rods with various aspect ratios $L / D$ as functions of the scaled volume fraction $n L^{3}$ on $\log -\log$ scale. Here $n$ is the number density. The solid line is a linear fit based on the results for $L / D=68$.

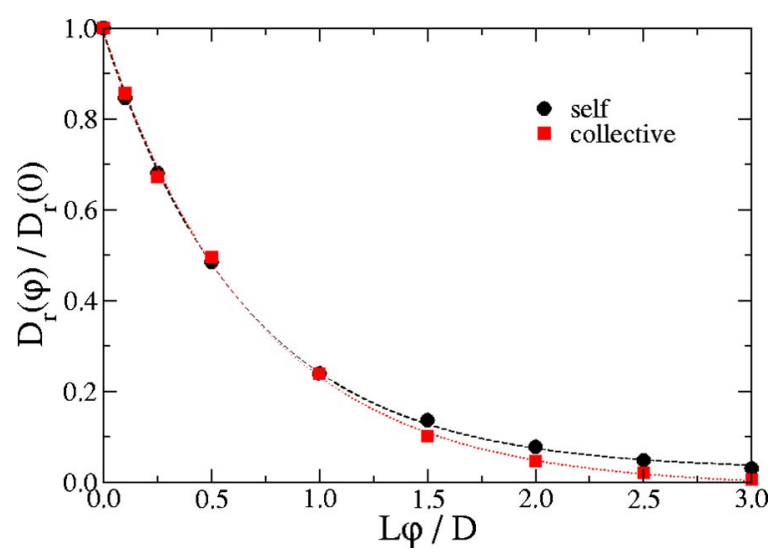

FIG. 5. The simulated self (circles) and collective (squares) rotational diffusion coefficients as functions of the scaled volume fraction $L \varphi / D$ for solutions of rods with $L / D=50$.

diffusion constants are almost equal to each other. At higher volume fractions, however, the collective diffusion coefficient keeps decaying, while the self-diffusion coefficient levels off to remain nonzero.

\section{B. Spinodals}

As a result of the severe constraints set by the infinitely hard interactions between the particles, dense systems of rods undergo a phase transition from an isotropic state to a nematic state. With increasing volume fractions, the orientations of the individual rods in the isotropic state become more correlated, until at the isotropic-nematic spinodal (INS) the isotropic state becomes absolutely unstable. At volume fractions above the INS, the slightest perturbation will drive an isotropic system towards the ordered nematic state. Similarly, on lowering the volume fraction, an initially stable nematic state will finally become unstable at the nematicisotropic spinodal (NIS) volume fraction. Actually no ordered state exists below the NIS, not even an unstable one. ${ }^{6}$ Between the NIS and INS, the fate of the system depends on its initial state. Initial states with enough order will end up in the nematic state, others will end up in the isotropic state. In the Onsager limit of infinitely long rods, the above scenario has been described in detail by Kayser and Raveche. ${ }^{6}$ For completeness we notice that the thermodynamically stable state is only obtained after the system has splitted into two different phases whose densities are given by the two binodal points.

In Fig. 6, we show the measured order parameter $P_{2}$ against the scaled volume fraction $L \varphi / D$ for solutions of rods with an aspect ratio of $L / D=20 . P_{2}$ is taken to be the stable value when the system has reached equilibrium starting from a predescribed state. The solid circles present order parameters of initially perfectly aligned systems decaying to the nematic or isotropic state. For dense systems when $L \varphi / D>3.0$, the order parameter $P_{2}$ decays from unity to some finite value indicative of the nematic state. In the cases when the volume fraction $L \varphi / D<3.0$, the nematic state becomes unstable and the order parameters $P_{2}$ of these systems 


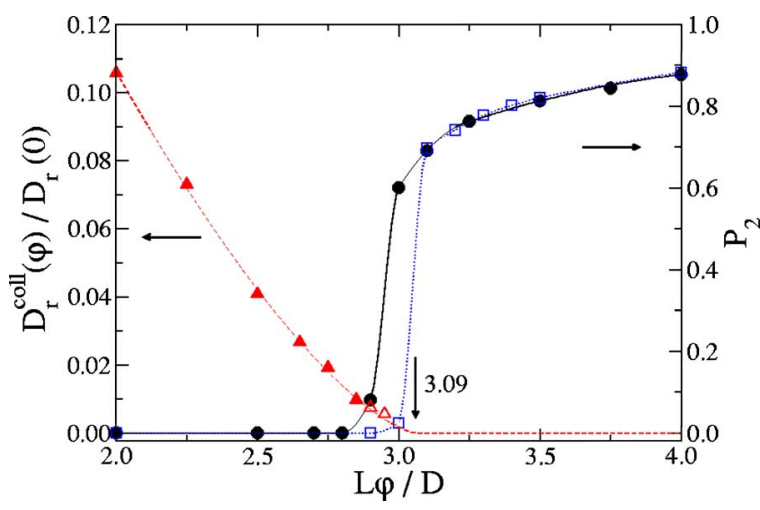

FIG. 6. The scalar order parameter $P_{2}$ vs the scaled volume fraction $L \varphi / D$ for solutions of rods with an aspect ratio of $L / D=20$. The closed circles and open squares denote stationary order parameters obtained when starting the simulations with perfectly aligned and isotropic boxes, respectively. The collective rotational diffusion coefficients are plotted as triangles. The closed triangles are calculated from the decay of an initially aligned state; the open triangles are obtained by autocorrelating thermal fluctuations, see Eq. (28). The dashed line is a fit with Eq. (29), reaching zero at the isotropic-nematic spinodal indicated by an arrow.

quickly decay to zero. The NIS can be read from the abrupt decrease of the order parameter, which is considered to occur at 3.0.

In order to measure the INS concentration, we performed simulations of initially isotropic systems with various volume fractions $L \varphi / D$. In systems with $L \varphi / D<3.0$, the rods remained chaotic, while in cases with $L \varphi / D>3.1$, all systems spontaneously ordered into a nematic phase. The isotropic-nematic spinodal is therefore located somewhere between $L \varphi / D=3.0$ and 3.1. The results of these simulations are represented by the squares in Fig. 6 .

For those systems where the density is very close to the INS concentration, the order parameter becomes more and more sensitive against the volume fraction, so that it is difficult to measure an accurate spinodal concentration using this method. In order to better pinpoint the INS volume fraction, we suggest to calculate the collective rotational diffusion coefficient $D_{r}^{\text {coll }}(\varphi)$ and search for the volume fraction where it becomes zero. The rationale for this is that at the INS, the driving force for the system to become either isotropic or nematic is zero. In Fig. 6, besides the order parameters already discussed, we have plotted the collective rotational diffusion coefficients for the case $L / D=20$. The solid triangles are obtained by studying the late stages of decaying perturbations $\delta \mathbf{S}(t)$ of the isotropic state, while the open triangles result from applying Eq. (28). The dashed line results from a fit through these points according to

$$
\frac{D_{r}^{\mathrm{coll}}(\varphi)}{D_{r}(0)}=A\left(\frac{L}{D} \varphi_{\mathrm{INS}}-\frac{L}{D} \varphi\right)^{\alpha}
$$

where $\varphi_{\text {INS }}$ is the INS volume fraction. The fit is only applicable in a restricted interval of $L \varphi / D$ values leading to a value of $A$ different from $\left(L \varphi_{\text {INS }} / D\right)^{-\alpha}$.

To map out the complete set of spinodals, similar simulations were performed for systems with aspect ratios $L / D$ $=10,15,30$, and 60. The results are shown in Figs. 7(a)-7(d). We estimated the nematic-to-isotropic spinodals from the abrupt decay of the order parameter $P_{2}$, obtaining

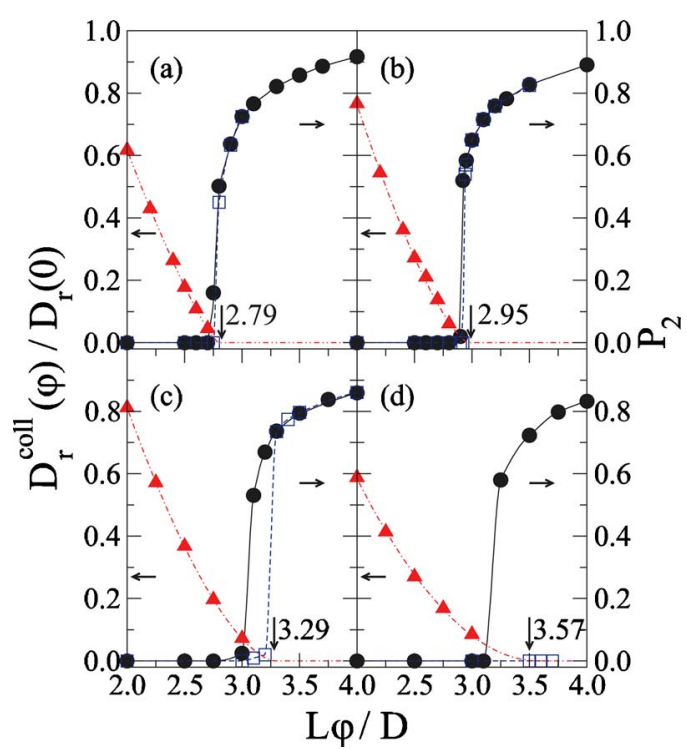

FIG. 7. The scalar order parameters $P_{2}$ and collective rotational diffusion coefficients $D_{r}^{\text {coll }}(\varphi)$ as functions of the scaled volume fraction $L \varphi / D$ for aspect ratios of 10 (a), 15 (b), 30 (c), and 60 (d). The arrows and numbers refer to the INS spinodals obtained by using Eq. (29). The values of $D_{r}^{\text {coll }}(\varphi) / D_{r}(0)$ in (a)-(d) are multiplied by $5,8,10$, and 20 , respectively.

$L \varphi_{\mathrm{NIS}} / D \approx 2.8,2.9,3.1$, and 3.25 for solutions of rods with $L / D=10,15,30$, and 60 , respectively. As with boxes with $L / D=20$, we calculated $D_{r}^{\text {coll }}(\varphi)$ in the stable and metastable isotropic states and calculated $\varphi_{\text {INS }}$ by fitting the results with Eq. (29). The measured $D_{r}^{\text {coll }}(\varphi)$ and the fits are shown in Figs. 7(a)-7(d). For curiosity we notice that the exponents in Eq. (29) are well described by

$$
\alpha=\frac{1}{\sqrt{2}}\left(\frac{L}{D}\right)^{1 / 4} .
$$

We summarize our findings about the spinodals of the isotropic-nematic phase transition by plotting the scaled volume fractions $L \varphi_{\mathrm{INS}} / D$ and $L \varphi_{\mathrm{NIS}} / D$ in Fig. 8 as functions of $D / L$. It is seen that the larger the aspect ratio is, the more discrepant are the volume fractions of the two spinodals,

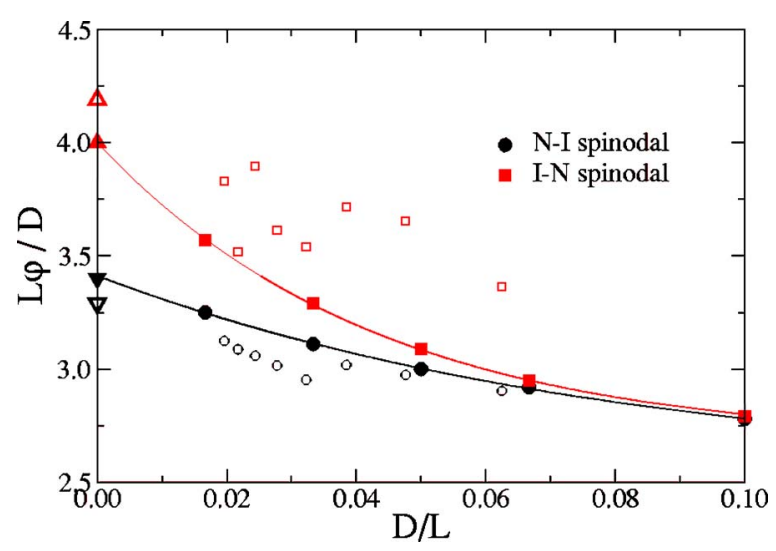

FIG. 8. The INS (solid squares) and NIS (solid circles) spinodals as functions of $D / L$. The open squares and circles are the binodals calculated in Ref. 19. The use of $D / L$, rather than the shape anisotropy $L / D$, facilitates the comparison with theoretical predictions. The theoretical binodals (open triangles) and spinodals (solid triangles) at infinite aspect ratio are plotted on the $y$ axis for $D / L=0$; these points were not included when fitting the lines. 
while for short rods the region between the two spinodals becomes quite narrow. The drawn lines in Fig. 8 are fits to the results of our simulations with

$$
\begin{aligned}
& L \varphi_{\mathrm{INS}} / D=1.4 e^{-23 D / L}+2.7, \\
& L \varphi_{\mathrm{NIS}} / D=0.9 e^{-12 D / L}+2.5 .
\end{aligned}
$$

Their extrapolations to the Onsager limit, i.e., to $D / L=0$, meet the vertical axis at $L \varphi_{\mathrm{NIS}} / D=3.4$ and $L \varphi_{\mathrm{INS}} / D=4.0$, respectively. These results are in good agreement with previous theoretical predictions. ${ }^{4,6}$ The open squares and circles in Fig. 8 are taken from Table XI in Bolhuis and Frenkel, ${ }^{19}$ and represent the binodals at the given values of $D / L$. It is seen that our spinodals are between the two binodals at all values of $D / L$, as they should be.

\section{Spinodal shift due to shear}

In shear flow, the director of a nematic system of rodlike colloids will orient itself along the flow direction. Besides this, the flow will slightly sharpen the orientational distribution of the individual rods and enlarge the order parameter. Also in the isotropic state an applied shear flow will slightly align the rods along the flow direction. As a result, the two spinodals corresponding to the isotropic-nematic phase transition will slightly shift to lower volume fractions with increasing shear rate. In accordance with our use in the previous section, we define $\varphi_{\text {INS }}$ and $\varphi_{\text {NIS }}$ as the volume fractions where

$$
\frac{d \mathbf{S}}{d t}=0, \quad \mathbf{S}(0)=\frac{1}{3} \hat{\mathbf{I}}
$$

and

$$
\frac{d \mathbf{S}}{d t}=0, \quad \mathbf{S}(0)=\hat{\mathbf{n}} \hat{\mathbf{n}},
$$

respectively, fail to have solutions close to $\mathbf{S}(0)$. According to Dhont and Briels ${ }^{23}$

$$
\begin{aligned}
\frac{d}{d t} \mathbf{S}= & -6 D_{r}\left\{\mathbf{S}-\frac{1}{3} \hat{\mathbf{I}}+\frac{5}{4} \frac{L}{D} \varphi\left(\mathbf{S}^{(4)}: \mathbf{S}-\mathbf{S} \cdot \mathbf{S}\right)\right\} \\
& +\dot{\gamma}\left\{\hat{\boldsymbol{\Gamma}} \cdot \mathbf{S}+\mathbf{S} \cdot \hat{\boldsymbol{\Gamma}}^{T}-2 \mathbf{S}^{(4)}: \hat{\mathbf{E}}\right\},
\end{aligned}
$$

where $\hat{\mathbf{E}}=\frac{1}{2}\left[\hat{\boldsymbol{\Gamma}}+\hat{\boldsymbol{\Gamma}}^{T}\right]$, with the superscript $T$ denoting a transposition, and $\mathbf{S}^{(4)}=\langle\hat{\mathbf{u}} \hat{u} \hat{u} \hat{\mathbf{u}}\rangle$. We added a factor of $5 / 4$ to the term proportional to $L \varphi / D$, which results from a slightly better evaluation of the excluded volume interaction between two rods with orientations $\hat{\mathbf{u}}$ and $\hat{\mathbf{u}}^{\prime}$, respectively (see Appendix A). The above equation may be closed by using the approximation $^{23}$

$$
\begin{aligned}
\mathbf{S}^{(4)}: \mathbf{M} \approx & \frac{1}{5}\{\mathbf{S} \cdot \mathbf{M}+\mathbf{M} \cdot \mathbf{S}-\mathbf{S} \cdot \mathbf{S} \cdot \mathbf{M}-\mathbf{M} \cdot \mathbf{S} \cdot \mathbf{S} \\
& +2 \mathbf{S} \cdot \mathbf{M} \cdot \mathbf{S}+3 \mathbf{S S}: \mathbf{M}\},
\end{aligned}
$$

where $\mathbf{M}$ is either $\mathbf{S}$ or $\hat{\mathbf{E}}$. Finally, $\boldsymbol{\Gamma}$ defines the flow by $\mathbf{V}=\boldsymbol{\Gamma} \cdot \mathbf{r}$ and equals $\dot{\gamma} \hat{\mathbf{e}}_{x} \hat{\mathbf{e}}_{y}$ for simple shear flow. Assuming uniaxial ordering and $\dot{\gamma}=0$, Eq. (33) may be solved analytically to find (see Appendix B)

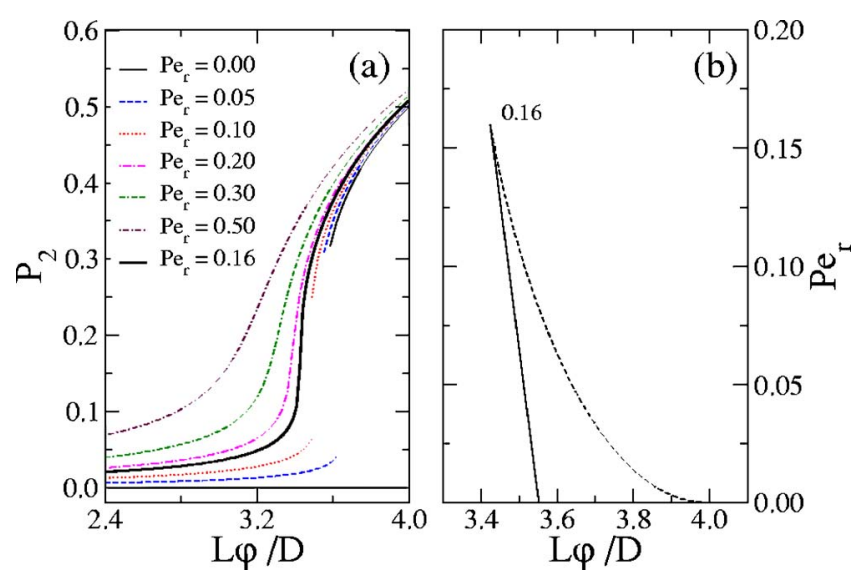

FIG. 9. (a) The theoretical scalar order parameter $P_{2}$ as a function of the scaled volume fraction $L \varphi / D$ at various shear rates for $L / D=\infty$. At low shear rates, the flow induces a small paranematic alignment in the isotropic phase and increases the alignment of the nematic phase. The end points of these lines are the INS and NIS spinodals, which are plotted in (b) as a function of the Peclet number $\mathrm{Pe}_{r}$. At the critical shear rate, corresponding to $\mathrm{Pe}_{r}=0.16$, the spinodals coalesce and end; hence the two phases merge into a single phase.

$$
P_{2}=\frac{3}{4} \sqrt{1-\frac{32}{9} \frac{D}{L \varphi}}+\frac{1}{4} .
$$

The results of solving Eqs. (32) and (33) are presented in Fig. 9. It is seen that with increasing Peclet number $\mathrm{Pe}_{r}=\dot{\gamma} / D_{r}$ the two spinodal volume fractions approach each other to become equal at the critical Peclet number $\mathrm{Pe}_{r}=0.16$, where the isotropic-nematic phase transition ceases to exist. ${ }^{23}$ For $\mathrm{Pe}_{r}=0$ the spinodals are $L \varphi_{\text {INS }} / D=4.0$ and $L \varphi_{\mathrm{NIS}} / D=32 / 9$ in good agreement with our finding in the previous section.

In Fig. 10 we present the results obtained by solving Eqs. (32) and (33) by means of Brownian dynamics simulations for $L / D=20$ and 30 and for various values of the Peclet number. Closed symbols refer to stationary states obtained by starting with $\mathbf{S}(0)=\hat{\mathbf{n}} \hat{\mathbf{n}}$, i.e., by solving Eq. (33), while open symbols refer to stationary states obtained by starting with $\mathbf{S}(0)=\frac{1}{3} \hat{\mathbf{I}}$. Obviously, the aspect ratios pertaining to this figure are all rather small for the results to apply to the case $L / D=\infty$ treated by the theory presented above. Unfortunately, it is extremely difficult to extend these simulations to systems with large aspect ratios. Still, from the results found so far, it is clear that theory and simulations are in agreement qualitatively. The order parameter is underestimated substantially by the theory, but the evolution of the spinodals with increasing Peclet number is qualitatively right. Moreover, the estimate of 0.16 for the critical Peclet number is well in the range $(0.1,0.2)$ found from the simulations.

\section{CONCLUSION}

We have described a new event-driven Brownian dynamics algorithm with which concentrated solutions of rigid spherocylinders in shear flow can be investigated. Compared with existing Brownian dynamics simulations of rigid rods where overlaps between particles are prevented by a repulsive potential, the novelty of the new code is in the fact that its time evolution is controlled by collisions, like in time of 


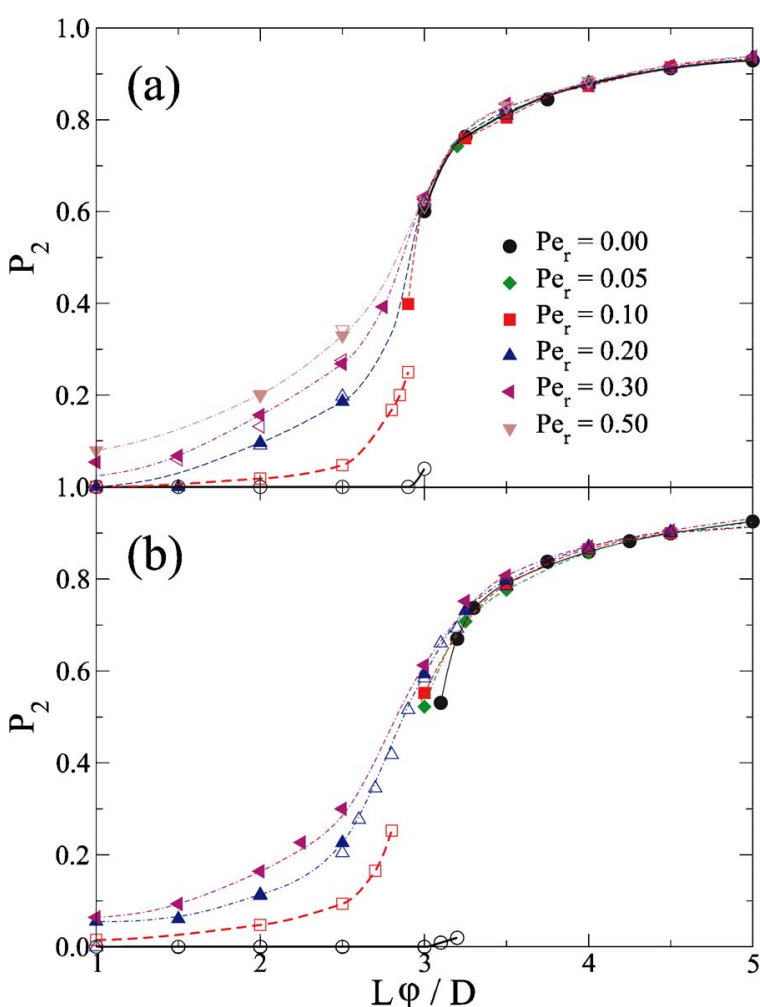

FIG. 10. The simulated scalar order parameters $P_{2}$ as functions of the scaled volume fraction $L \varphi / D$ at various shear rates for (a) $L / D=20$ and (b) $L / D$ $=30$.

flight molecular dynamics simulations. Excluded volume interactions between the rods are considered to be infinitely hard, but hydrodynamic interactions are neglected. The new algorithm is very efficient, as exemplified by the fact that the time step $\delta t$ is ten times larger than the one used in our previous work. ${ }^{24}$ The general picture of the present paper is summarized as follows: (i) The calculated rotational selfdiffusion coefficients $D_{r}(\varphi)$ by both the new event-driven Brownian dynamics simulations and our previous program accord very well with each other, confirming the validity of our new simulation algorithm. (ii) Isotropic-nematic spinodals are calculated of rod systems with various aspect ratios $L / D$. The simulated INS and NIS spinodals are well located between the binodals measured by Bolhuis and Frenkel ${ }^{19}$ by Monte Carlo simulations, as they should be. The extrapolations of the spinodals to the infinitely long rod system are in good agreement with previous theoretical predictions by Onsager and Kayser et al. (iii) Shear shifts of the spinodals to lower values are investigated. The measured order parameters deviate substantially from the theoretical predictions, ${ }^{23}$ but the spinodal concentrations agree rather well. The estimated critical rotational Peclet number $\mathrm{Pe}_{r}$, above which only continuous and reversible changes of the order parameters can be found, agree qualitatively with the predicted value $\mathrm{Pe}_{r}=0.16$.

The present successful Brownian dynamics algorithm allows us to investigate in detail the dynamical properties of nematic liquid-crystalline solutions of rigid spherocylinders in shear flow. Some fascinating rheological phenomena such as the oscillating responses of the director, i.e., tumbling, kayaking, wagging, or flow-aligning, to a steady driving "force" will be published separately.

\section{ACKNOWLEDGMENT}

This work is part of the SoftLink research program of the Stichting voor Fundamenteel Onderzoek der Materie (FOM), which is financially supported by the Nederlandse Organisatie voor Wetenschappelijk Onderzoek (NWO).

\section{APPENDIX A: MAIER-SAUPE EXPANSION}

In the derivation of Eq. (34), the excluded volume of two rigid rods gives rise to an effective potential proportional to

$$
\int d^{3} \mathbf{R} \chi\left(\mathbf{R}, \hat{\mathbf{u}}, \hat{\mathbf{u}}^{\prime}\right)=2 D L^{2}\left|\hat{\mathbf{u}} \times \hat{\mathbf{u}}^{\prime}\right|,
$$

where $\mathbf{R}$ is the vector separating two rods with orientations $\hat{\mathbf{u}}$ and $\hat{\mathbf{u}}^{\prime}$, respectively, and $\chi\left(\mathbf{R}, \hat{\mathbf{u}}, \hat{\mathbf{u}}^{\prime}\right)$ equals 1 when two rods overlap and 0 otherwise. The vector products $\left|\hat{\mathbf{u}} \times \hat{\mathbf{u}}^{\prime}\right|$ may be expanded in terms of polyadic products $\mathbf{q}_{k}$ of $\hat{\mathbf{u}}$ 's, which may be chosen such that

$$
\oint d \hat{\mathbf{u}} \mathbf{q}_{k} \mathbf{q}_{l} \sim \delta_{k, l} \text {. }
$$

The first three of these may be chosen as

$$
\begin{aligned}
& \mathbf{q}_{0}=1, \\
& \mathbf{q}_{1}=\hat{\mathbf{u}}, \\
& \mathbf{q}_{2}=\hat{\mathbf{u}} \hat{\mathbf{u}}-\frac{1}{3} \hat{\mathbf{I}} .
\end{aligned}
$$

Expanding $\left|\hat{\mathbf{u}} \times \hat{\mathbf{u}}^{\prime}\right|$ up to second order, we obtain

$$
\left|\hat{\mathbf{u}} \times \hat{\mathbf{u}}^{\prime}\right|=a_{0}+a_{2} \mathbf{q}_{2}^{\prime}: \mathbf{q}_{2},
$$

where we have used the existing symmetry between $\hat{\mathbf{u}}$ and $\hat{\mathbf{u}}^{\prime}$. Using

$$
\begin{aligned}
& \oint d \hat{\mathbf{u}}\left|\hat{\mathbf{u}} \times \hat{\mathbf{u}}^{\prime}\right|=\pi^{2}, \\
& \oint d \hat{\mathbf{u}}\left|\hat{\mathbf{u}} \times \hat{\mathbf{u}}^{\prime}\right| \mathbf{q}_{2}=-\frac{\pi^{2}}{8} \mathbf{q}_{2}^{\prime},
\end{aligned}
$$

we obtain

$$
\begin{aligned}
& a_{0}=\frac{1}{4} \pi, \\
& a_{2}=-\frac{15}{64} \pi .
\end{aligned}
$$

Using Eq. (A6) in Eq. (A4) and rewriting the result a bit we arrive at 


$$
\left|\hat{\mathbf{u}} \times \hat{\mathbf{u}}^{\prime}\right|=\frac{21 \pi}{64}\left[1-\frac{5}{7} \hat{\mathbf{u}} \hat{\mathbf{u}}: \hat{\mathbf{u}}^{\prime} \hat{\mathbf{u}}^{\prime}\right] .
$$

Compared with the corresponding expansion in Ref. 23, the second term has an extra factor of $5 / 4$, which finally leads to the factor of $5 / 4$ in front of the term proportional to $L \varphi / D$ in Eq. (34).

\section{APPENDIX B: ZERO SHEAR NEMATIC ORDER PARAMETER}

For the case when $\dot{\gamma}=0$ the equation of motion of $\mathbf{S}$ simplifies to

$$
\frac{d}{d t} \mathbf{S}=-6 D_{r}\left\{\mathbf{S}-\frac{1}{3} \hat{\mathbf{I}}+\frac{3}{4} \frac{L}{D} \varphi(\mathbf{S S}: \mathbf{S}-\mathbf{S} \cdot \mathbf{S})\right\},
$$

where we have made use of the closure equation (35). Assuming uniaxial symmetry, $\mathbf{S}$ may be written like

$$
\begin{aligned}
\mathbf{S} & =\lambda \hat{\mathbf{n}} \hat{\mathbf{n}}+\frac{1}{2}(1-\lambda) \hat{\mathbf{e}}_{2} \hat{\mathbf{e}}_{2}+\frac{1}{2}(1-\lambda) \hat{\mathbf{e}}_{3} \hat{\mathbf{e}}_{3} \\
& =\frac{1}{2}(1-\lambda) \hat{\mathbf{I}}+\frac{1}{2}(3 \lambda-1) \hat{\mathbf{n}} \hat{\mathbf{n}},
\end{aligned}
$$

where $\hat{\mathbf{e}}_{2}$ and $\hat{\mathbf{e}}_{3}$ are two unit vectors perpendicular to each other and to $\hat{\mathbf{n}}$. Introducing this into Eq. (B1), one finds after some rewriting

$$
\frac{d}{d t}\left[\mathbf{S}-\frac{1}{3} \hat{\mathbf{I}}\right]=-6 D_{r}\left\{1-\frac{9}{8} \frac{L}{D} \varphi \lambda(1-\lambda)\right\}\left[\mathbf{S}-\frac{1}{3} \hat{\mathbf{I}}\right] .
$$

A stationary nonzero value of $\mathbf{S}-\frac{1}{3} \hat{\mathbf{I}}$ can only be found for values of $L \varphi / D$ for which the factor between curly brackets can be put equal to zero. This leads to Eq. (36).

${ }^{1}$ T. A. Lenstra, Z. Dogic, and J. K. G. Dhont, J. Chem. Phys. 114, 10151 (2001).

${ }^{2}$ M. P. Lettinga and J. K. G. Dhont, J. Phys.: Condens. Matter 16, S3929 (2004).

${ }^{3}$ M. P. Lettinga, K. Kang, A. Imhof, D. Derks, and J. K. G. Dhont, J. Phys.: Condens. Matter 17, S3609 (2005).

${ }^{4}$ L. Onsager, Ann. N.Y. Acad. Sci. 51, 627 (1949).

${ }^{5}$ G. Lasher, J. Chem. Phys. 53, 4141 (1970).

${ }^{6}$ R. F. Kayser, Jr. and H. J. Raveché, Phys. Rev. A 17, 2067 (1978).

${ }^{7}$ H. N. W. Lekkerkerker, P. Coulon, R. van der Haegen, and R. Deblieck, J. Chem. Phys. 80, 3427 (1984).

${ }^{8}$ T. Shimada, M. Doi, and K. Okano, J. Chem. Phys. 88, 7181 (1988).

${ }^{9}$ S. Varga, G. Jackson, and I. Szalai, Mol. Phys. 93, 377 (1998).

${ }^{10}$ J. K. G. Dhont, M. P. B. van Bruggen, and W. J. Briels, Macromolecules 32, 3809 (1999).

${ }^{11}$ E. Garcia, D. C. Williamson, and A. Martinez-Richa, Mol. Phys. 98, 179 (2000).

${ }^{12}$ K. Shundyak and R. van Roij, J. Phys.: Condens. Matter 13, 4789 (2001).

${ }_{13}^{13}$ A. Speranza and P. Sollich, J. Chem. Phys. 117, 5421 (2002).

${ }^{14}$ A. Speranza and P. Sollich, Phys. Rev. E 67, 061702 (2003).

${ }^{15}$ K. Shundyak and R. van Roij, Phys. Rev. E 69, 041703 (2004).
${ }^{16}$ R. van Roij, Eur. J. Phys. 26, S57 (2005).

${ }^{17}$ Z. Dogic and S. Fraden, Philos. Trans. R. Soc. London, Ser. A 359, 997 (2001).

${ }^{18}$ Z. Dogic, K. Purdy, E. Grelet, M. Adams, and S. Fraden, Phys. Rev. E 69, 051702 (2004).

${ }^{19}$ P. G. Bolhuis and D. Frenkel, J. Chem. Phys. 106, 666 (1997).

${ }^{20}$ P. G. Bolhuis, A. Stroobants, D. Frenkel, and H. N. W. Lekkerkerker, J. Chem. Phys. 107, 1551 (1997).

${ }^{21}$ G. Germono and F. Schmid, J. Chem. Phys. 123, 214703 (2005).

${ }^{22}$ G. J. Vroege and H. N. W. Lekkerkerker, Rep. Prog. Phys. 55, 1241 (1992).

${ }^{23}$ J. K. G. Dhont and W. J. Briels, Colloids Surf., A 213, 131 (2003).

${ }^{24}$ Y.-G. Tao, W. K. den Otter, J. T. Padding, J. K. G. Dhont, and W. J. Briels, J. Chem. Phys. 122, 244903 (2005).

${ }^{25}$ P. D. Cobb and J. E. Butler, J. Chem. Phys. 123, 054908 (2005).

${ }^{26}$ H. See, M. Doi, and R. Larson, J. Chem. Phys. 92, 792 (1990).

${ }^{27}$ P. D. Olmsted and P. M. Goldbart, Phys. Rev. A 46, 4966 (1992).

${ }^{28}$ P. D. Olmsted and C.-Y. D. Lu, Phys. Rev. E 60, 4397 (1999).

${ }^{29}$ G. Rienäcker and S. Hess, Physica A 267, 294 (1999).

${ }^{30}$ J. K. G. Dhont and W. J. Briels, J. Chem. Phys. 117, 3992 (2002).

${ }^{31}$ J. K. G. Dhont and W. J. Briels, J. Chem. Phys. 118, 1466 (2003).

${ }^{32}$ J. K. G. Dhont and W. J. Briels, Phys. Rev. E 72, 031404 (2005).

${ }^{33}$ M. Doi and S. F. Edwards, The Theory of Polymer Dynamics (Clarendon, Oxford, 1986).

${ }^{34}$ J. K. G. Dhont, An Introduction to Dynamics of Colloids (Elsevier, Amsterdam, 1996).

${ }^{35}$ W. J. Briels, Theory of Polymer Dynamics, Lecture notes (Uppsala University, Uppsala, 1994).

${ }^{36}$ D. C. Rapaport, The Art of Molecular Dynamics Simulation, 2nd ed. (Cambridge University Press, Cambridge, 2004).

${ }^{37}$ M. P. Allen and D. J. Tildesley, Computer Simulation of Liquids (Clarendon, Oxford, 1987).

${ }^{38}$ D. Frenkel and J. F. Maguire, Mol. Phys. 49, 503 (1983).

${ }^{39}$ E. A. J. Peters and T. M. A. O. M. Barenbrug, Phys. Rev. E 66, 056701 (2002).

${ }^{40}$ E. A. J. Peters and T. M. A. O. M. Barenbrug, Phys. Rev. E 66, 056702 (2002).

${ }^{41}$ T. M. A. O. M. Barenbrug, E. A. J. Peters, and J. D. Schieber, J. Chem. Phys. 106, 8624 (1997).

${ }^{42}$ B. Cichocki and K. Hinsen, Physica A 166, 473 (1990).

${ }^{43}$ D. M. Heyes and J. R. Melrose, J. Non-Newtonian Fluid Mech. 46, 1 (1993).

${ }^{44}$ W. Schaertl and H. Sillescu, J. Stat. Phys. 74, 687 (1994).

${ }^{45}$ I. Moriguchi, K. Kawasaki, and T. Kawakastu, J. Phys. II 5, 143 (1995).

${ }^{46}$ I. Moriguchi, J. Chem. Phys. 117, 9202 (1997).

${ }^{47}$ P. Strating, Phys. Rev. E 59, 2175 (1999).

${ }^{48}$ M. Doi, I. Yamamoto, and F. Kano, J. Phys. Soc. Jpn. 53, 3000 (1984).

${ }^{49}$ O. G. Harlen, R. R. Sundararajakumar, and D. L. Koch, J. Fluid Mech. 388, 355 (1999).

${ }^{50}$ Y.-G. Tao, W. K. den Otter, and W. J. Briels, Phys. Rev. Lett. 95, 237802 (2005).

${ }^{51}$ Y.-G. Tao, W. K. den Otter, and W. J. Briels, J. Chem. Phys. (to be published).

${ }^{52}$ S. R. Williams and A. P. Philipse, Phys. Rev. E 67, 051301 (2003).

${ }^{53}$ M. Doi and S. F. Edwards, J. Chem. Soc., Faraday Trans. 2 74, 560 (1978).

${ }^{54}$ M. Doi and S. F. Edwards, J. Chem. Soc., Faraday Trans. 2 74, 918 (1978).

${ }^{55}$ D. Chandler, Introduction to Modern Statistical Mechanics (Oxford University Press, New York, 1987).

${ }^{56}$ W. J. Briels, Theory of Simple Liquids, Lecture notes (Uppsala University, Uppsala, 1993). 